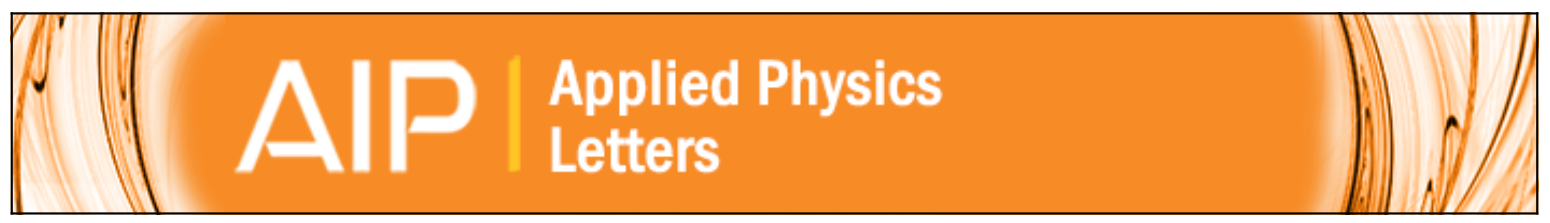

\title{
Aluminum doped zinc oxide for organic photovoltaics
}

G. B. Murdoch, S. Hinds, E. H. Sargent, S. W. Tsang, L. Mordoukhovski, and Z. H. Lu

Citation: Applied Physics Letters 94, 213301 (2009); doi: 10.1063/1.3142423

View online: http://dx.doi.org/10.1063/1.3142423

View Table of Contents: http://scitation.aip.org/content/aip/journal/apl/94/21?ver=pdfcov

Published by the AIP Publishing

\section{Articles you may be interested in}

Properties of solid solutions, doped film, and nanocomposite structures based on zinc oxide

Low Temp. Phys. 41, 129 (2015); 10.1063/1.4908204

Influence of growth temperature on electrical, optical, and plasmonic properties of aluminum:zinc oxide films grown by radio frequency magnetron sputtering

J. Appl. Phys. 114, 143506 (2013); 10.1063/1.4824751

Doped zinc oxide window layers for dye sensitized solar cells

J. Appl. Phys. 114, 134506 (2013); 10.1063/1.4824363

Aluminum-doped zinc oxide formed by atomic layer deposition for use as anodes in organic light emitting diodes

J. Vac. Sci. Technol. A 31, 01A101 (2013); 10.1116/1.4738749

Deposition of aluminum-doped zinc oxide thin films for optical applications using if and dc magnetron sputter deposition

J. Vac. Sci. Technol. A 28, 515 (2010); 10.1116/1.3425640

\section{AIP $\mid$ APL Photonics}

APL Photonics is pleased to announce Benjamin Eggleton as its Editor-in-Chief

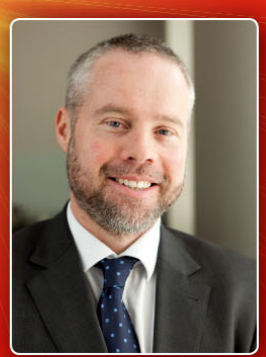




\title{
Aluminum doped zinc oxide for organic photovoltaics
}

\author{
G. B. Murdoch, ${ }^{\text {a) }}$ S. Hinds, E. H. Sargent, S. W. Tsang, L. Mordoukhovski, and Z. H. Lu ${ }^{\text {b) }}$ \\ Department of Materials Science and Engineering, University of Toronto, 184 College St., Toronto, \\ Ontario M5S 3E4, Canada
}

(Received 14 January 2009; accepted 22 April 2009; published online 29 May 2009)

\begin{abstract}
Aluminum doped zinc oxide (AZO) was grown via magnetron sputtering as a low-cost alternative to indium tin oxide (ITO) for organic photovoltaics (OPVs). Postdeposition ozone treatment resulted in devices with lower series resistance, increased open-circuit voltage, and power conversion efficiency double that of devices fabricated on untreated AZO. Furthermore, cells fabricated using ozone treated AZO and standard ITO displayed comparable performance. (C) 2009 American Institute of Physics. [DOI: 10.1063/1.3142423]
\end{abstract}

Organic photovoltaics (OPVs) have drawn increasing interest as a route to capture sunlight for low-cost renewable energy. Since Tang ${ }^{1}$ introduced the first donor-acceptor (DA) device more than a decade ago, power conversion efficiencies of OPVs have steadily risen, reaching efficiencies higher than $5 \%$ for small-molecule devices. ${ }^{2}$ Polymer-based photovoltaics show similar performance gains. ${ }^{3}$ All photovoltaics, including OPVs, require the use of transparent conducting electrode materials in order to admit light into the active region as well as to collect charge. The cost of transparent conductive films is thus a critical component of the total OPV price. Indium tin oxide (ITO) is currently the de facto standard transparent conductive oxide (TCO) used in OPV technology due its high optical transparency, metallic conductivity, and high work function. Relatively little investigation has occurred involving the use of alternative TCOs for small-molecule OPV applications. This work is essential, however, given that ITO has become approximately ten times more expensive over the past 5 years due to diminishing indium resources; ${ }^{4}$ the cost of indium is only expected to grow with increasing demand from producers of photovoltaics on top of the existing flat-panel display industry.

The properties of $\mathrm{ZnO}$ doped with group three elements such as $\mathrm{Al}$ or Ga have been extensively investigated for use as low-cost TCOs. $\mathrm{ZnO}$ is a wide bandgap $(3.3 \mathrm{eV}), n$-type semiconductor that can be produced by methods including pulsed laser deposition, chemical vapor deposition, spray pyrolysis, and magnetron sputtering. ${ }^{5} \mathrm{ZnO}$ doped with aluminum (AZO) is well suited to PV applications owing to its high electrical conductivity and excellent optical transparency in the near infrared and visible regions. ITO-equivalent performance for phthalocyanine-based OPV devices fabricated on $\mathrm{ZnO}$ without the need for a buffer layer has not been demonstrated. ${ }^{4,6}$ One report of ITO-equivalent performance, however, has been made for oligothiphene-based cells. $^{7}$ In this work, we show the importance of sputtering conditions and postdeposition ozone treatment of AZO prior to OPV fabrication. Furthermore, we show that ozone treated AZO is an effective ITO replacement for doubleheterojunction phthalocyanine-based devices.

OPV devices were fabricated on glass substrates cleaned in acetone, methanol, and de-ionized water, followed by

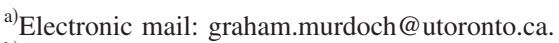

${ }^{b)}$ Electronic mail: zhenghong.lu@utoronto.ca.
}

15 min ultraviolet (UV)-ozone treatment. Reference devices were fabricated on ITO coated glass $(15 \Omega / \square)$ cleaned in the same manner. AZO films were produced using dc magnetron sputtering in a BOC Edwards Auto 306 coating system equipped with an MDX $1.5 \mathrm{~K}$ dc plasma generator power supply. The base pressure was $\sim 10^{-7}$ Torr. A 3 in. diameter $\mathrm{ZnO}$ target doped with 2 wt $\% \mathrm{Al}_{2} \mathrm{O}_{3}$ was sputtered at $50 \mathrm{~W}$ in a $5 \mathrm{mTorr} \mathrm{Ar}$ atmosphere. Substrates were placed at $8,12,16$, and $19 \mathrm{~cm}$ center-to-center distance from the target. The substrates were oriented perpendicular to the target and were offset horizontally from the target center by $6 \mathrm{~cm}$. Anode lines were defined by photolithography using Shipley S1813 photoresist and 2\% $\mathrm{HCl}$ etchant solution. Sheet resistance measurements of all films were taken using a Keithley 2400 SourceMeter and four-point probes. Film thicknesses were measured using a KLA Tencor P-16+ stylus profilometer. Optical transmission and reflection spectra were taken using a Cary spectrophotometer equipped with an integrating sphere. Surface morphology was observed via scanning electron microscopy (SEM) and atomic force microscopy (AFM) in tapping mode. AZO films were treated by ex situ oxidation for $60 \mathrm{~min}$ by UV-ozone treatment.

Metal and organic films were thermally evaporated in separate chambers with base pressures of $\sim 10^{-7}$ and $\sim 10^{-8}$ Torr, respectively. Film thicknesses were monitored using calibrated quartz crystal monitors. Organic solar cells were fabricated using copper phthalocyanine $(\mathrm{CuPc})$ and Buckminsterfullerene $\left(\mathrm{C}_{60}\right)$ as the DA system. The cell was finished using an exciton blocking layer of 1,3,5-tris (2-Nphenylbenzimidazolyl) benzene (TPBI) and an aluminum cathode. The final device structure was as follows: anode/ $\mathrm{CuPc}(30 \mathrm{~nm}) / \mathrm{C}_{60}(30 \mathrm{~nm}) / \mathrm{TPBI}(6 \mathrm{~nm}) / \mathrm{Al}(80 \mathrm{~nm})$. The active area was $1 \times 2 \mathrm{~mm}^{2}$ for all devices. Device current density versus voltage $(J-V)$ characteristics were measured using a Keithley 6430 subfemptoamp meter under $100 \mathrm{~mW} / \mathrm{cm}^{2}$ simulated AM1.5G solar illumination.

Figure 1(a) shows optical transmission spectra for untreated AZO films deposited at target-to-substrate distances of $8,12,16$, and $19 \mathrm{~cm}$. Figure 1(b) shows electrical resistivity of AZO films as a function of target-to-substrate distance for as-deposited and ozone-treated samples. Film thicknesses were 98, 164, 248, and $541 \mathrm{~nm}$, respectively, owning to varying deposition rates within one deposition. These thicknesses were accounted for in the calculation of bulk resistivity. High transparency is observed throughout 

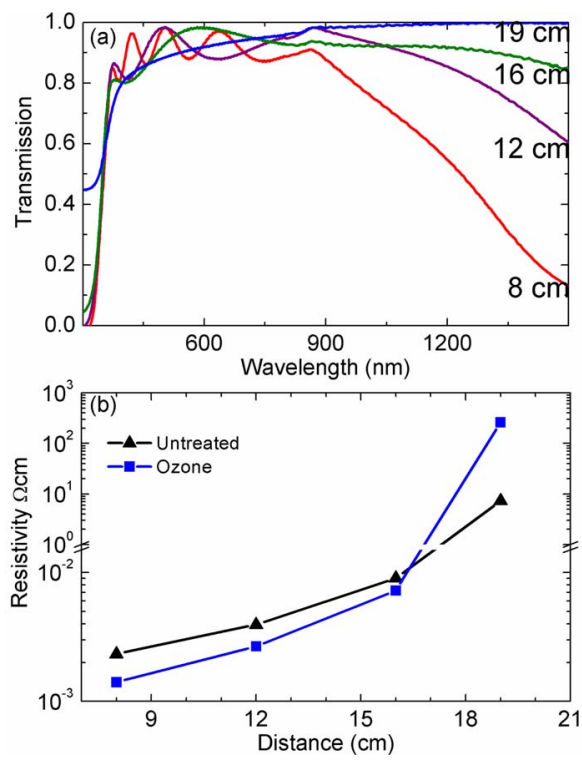

FIG. 1. (Color online) (a) Optical transmission vs wavelength for untreated, dc sputtered AZO films deposited at 8,12,16, and $19 \mathrm{~cm}$ target-to-substrate distances. (b) Film resistivity for as-deposited and ozone treated AZO vs target-to-substrate distance.

the visible spectrum with an average transmission of $92 \%$ between 380 and $750 \mathrm{~nm}$ for the film deposited at $8 \mathrm{~cm}$. With decreasing target-to-substrate distance, the absorption edge of the AZO films moves toward lower wavelengths. We therefore infer by the Drude model ${ }^{5}$ that shorter target-tosubstrate distance resulted in higher free carrier concentration for AZO films deposited in our system.

Film resistivity increased monotonically with increasing target-to-substrate distance, as expected from optical measurements of carrier concentration. Significantly, ozone treatment lowered the resistivities of the most conductive AZO films. The resistivity of the film deposited at $8 \mathrm{~cm}$ was decreased by $40 \%$ from $2.3 \times 10^{-3}$ to $1.4 \times 10^{-3} \Omega \mathrm{cm}$. At a distance of $19 \mathrm{~cm}$ from the target, the resistivity of asdeposited films increased abruptly by approximately three orders of magnitude. Interestingly, these films became much more resistive after ozone treatment. SEM images of asdeposited AZO films are shown in Fig. 2. With increasing target-to-substrate distance, grain sizes became progressively smaller. A grain size of approximately $50 \mathrm{~nm}$ was observed for the most conductive film deposited at $8 \mathrm{~cm}$. The surface roughness of this film was found to be $6 \mathrm{~nm}$ by AFM. Increased conductivity at shorter range is attributable to in-

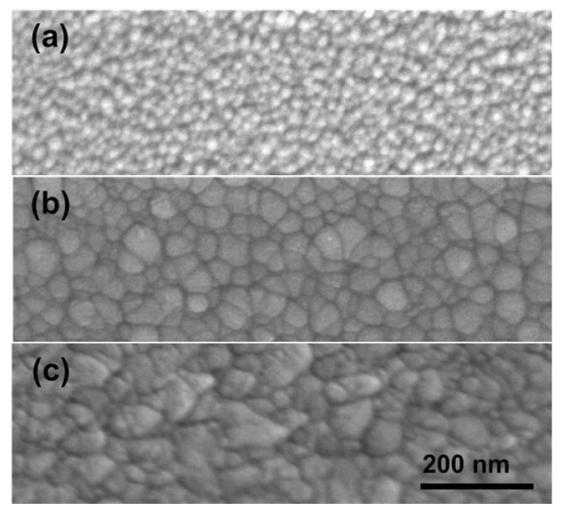

FIG. 2. SEM micrographs of AZO films grown at target-to-substrate distances of (a) $19 \mathrm{~cm}$, (b) $16 \mathrm{~cm}$, and (o) $8 \mathrm{~cm}$.



FIG. 3. (Color online) (a) Current density and (b) photocurrent density vs voltage for OPV devices made using as-deposited and ozone treated AZO.

creased carrier mobility (as expected from larger grain size and decreased grain boundary scattering), in addition to the higher carrier concentration observed optically. Since doping in $\mathrm{AZO}$ is thought to arise from $\mathrm{Al}$ substitution on $\mathrm{Zn}$ cationic sites, ${ }^{8}$ we posit that the increase in conductivity with UV-ozone treatment for the most conductive films was due to a reduction in the number of oxygen vacancies in the AZO lattice, leading to reduced impurity scattering and higher electron mobility. No observable change in grain structure was seen by SEM after UV-ozone treatment. Lower carrier concentration with target-to-substrate distance indicates less $\mathrm{Al}$ incorporation into the $\mathrm{ZnO}$ lattice and subsequent segregation to grain boundaries or incorporation into $\mathrm{Al}_{2} \mathrm{O}_{3}$. For the least conductive film, the observed increase in resistivity with UV-ozone treatment was likely due to the reaction of segregated aluminum with oxygen, producing insulating $\mathrm{Al}_{2} \mathrm{O}_{3}$.

Double heterostructure cells were prepared on the highest conductivity as-deposited (device A) and UV-ozone treated (device B) AZO films in a single deposition sequence. The sheet resistances of these films were 43 and $26 \Omega / \square$, respectively. Figure 3(a) shows the current-voltage characteristics of both types of device under $100 \mathrm{~mW} / \mathrm{cm}^{2}$ simulated solar illumination. A noticeable change in device performance occurred following ozone treatment. Cell B exhibited higher open circuit voltage $\left(V_{\mathrm{OC}}\right)$ and lower series resistance. A power conversion efficiency of $1.3 \%$ was attained for device B as compared to $0.7 \%$ for device A. The forward bias dark current of sample B was increased and the

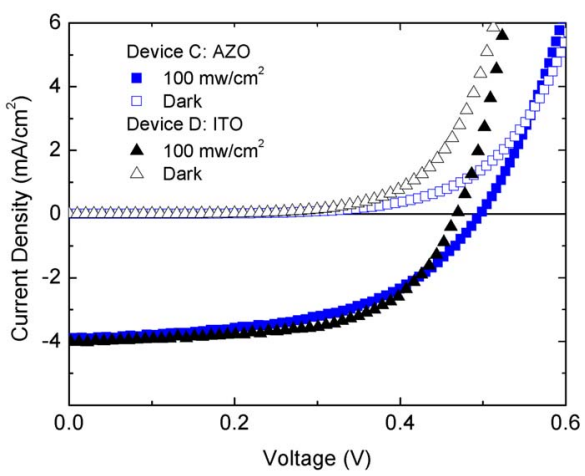

FIG. 4. (Color online) Current density at $100 \mathrm{~mW} / \mathrm{cm}^{2}$ and dark illumination levels ys voltage for OPV cells. 
TABLE I. Open circuit voltage $V_{\mathrm{OC}}$, short circuit current density $J_{\mathrm{SC}}$, FF, power conversion efficiency $\eta_{P}$, and the calculated series resistance $R_{S}$ of all cells.

\begin{tabular}{cccccccc}
\hline \hline \multirow{2}{*}{ Experiment } & Device & Anode & $\begin{array}{c}V_{\mathrm{OC}} \\
(\mathrm{V})\end{array}$ & $\begin{array}{c}J_{\mathrm{SC}} \\
\left(\mathrm{mA} / \mathrm{cm}^{2}\right)\end{array}$ & FF & $\begin{array}{c}\eta_{P} \\
(\%)\end{array}$ & $\begin{array}{c}R_{S} \\
\left(\Omega \mathrm{cm}^{2}\right)\end{array}$ \\
\hline \multirow{2}{*}{1} & $\mathrm{~A}$ & $\mathrm{AZO}$ & 0.42 & 4.46 & 0.37 & 0.7 & 65 \\
\multirow{2}{*}{2} & $\mathrm{~B}$ & $\mathrm{AZO}\left(\mathrm{O}_{3}\right)$ & 0.50 & 4.53 & 0.55 & 1.3 & 19 \\
& $\mathrm{C}$ & $\mathrm{AZO}\left(\mathrm{O}_{3}\right)$ & 0.49 & 3.96 & 0.53 & 1.0 & 24 \\
& $\mathrm{D}$ & ITO & 0.46 & 4.04 & 0.60 & 1.1 & 18 \\
\hline
\end{tabular}

reverse bias current was decreased, indicating a reduced hole injection barrier and a larger barrier to electron injection for ozone treated AZO into CuPc. X-ray photoelectron spectroscopy secondary cutoff measurements indicate an increase in work function from $4.2 \mathrm{eV}$ as deposited to $4.7 \mathrm{eV}$ after in situ ozone treatment of unpatterned AZO.

Figure 3(b) shows photocurrent-voltage curves for devices $\mathrm{A}$ and $\mathrm{B}$ obtained by subtracting dark from light current densities. Ozone treatment resulted in a clear shift of the photocurrent characteristic toward higher bias $(\Delta V=0.2 \mathrm{~V})$. When taken with the aforementioned increase in forward bias dark current, this shift indicates an improved band alignment for ozone treated AZO, bringing the AZO Fermi level $0.2 \mathrm{eV}$ closer to CuPc's highest occupied molecular orbital upon contact. ${ }^{9}$ Consequently, device B has a larger built-in voltage $\left(V_{\mathrm{bi}}\right)$. Figure 3(c) schematically shows the energy level alignment for samples A and B under open circuit conditions. Using the Einstein relation, ${ }^{10}$ the sum of drift and diffusion currents for electrons can be written in terms of the gradient in quasi-Fermi level, $\nabla E_{F n}$,

$$
J_{n}(x)=e n(x) \mu_{n} \nabla E_{F n}(x),
$$

where $e$ is the charge of an electron, $n(x)$ is the carrier concentration, and $\mu_{n}$ is the electron mobility. ${ }^{11}$ A similar relation applies for holes. At $V_{\mathrm{OC}}$, zero net current flows as drift balances diffusion and the quasi-Fermi levels $E_{F n}$ and $E_{F p}$ are constant from the DA interface to the contacts, as shown in Fig. 3(c). Furthermore, assuming no voltage drop across the contact interfaces, $V_{\mathrm{OC}}$ equals the maximum splitting of the quasi-Fermi levels across the solar cell, ${ }^{11}$

$$
V_{\mathrm{OC}}=E_{F n}-E_{F p} \text {. }
$$

The amount of splitting rises as the free carrier concentration in each layer rises. The electric field developed across the organics due to quasi-Fermi-level splitting under illumination counteracts the built-in field. The net potential gradient across the organics is thus the difference between $V_{\mathrm{OC}}$ and $V_{\mathrm{bi}}{ }^{12}$ This net potential gradient, which tends to confine free carriers to the DA interface, must be smaller in device B due to its larger $V_{\mathrm{bi}}$, as shown in Fig. 3(c). Less interface recombination due to reduced carrier accumulation at the DA interface and improved exciton dissociation are therefore expected for device $\mathrm{B}$. The resulting increase in free carrier concentration in device B leads to higher photocurrent close $V_{\mathrm{OC}}$, as well as an increase in $V_{\mathrm{OC}}$ itself due to greater quasiFermi-level splitting. ${ }^{12}$

A sigmoidal $J-V$ characteristic was observed for device A but not for device B. Approaching $0.4 \mathrm{~V}$, the electric field in the organics begins to oppose the flow of photogenerated free carriers, causing carrier accumulation at the at the DA interface. As mentioned, this effect is more pronounced for device A due its smaller built-in potential. Higher carrier concentration at the DA interface increases recombination and lowers the observed photocurrent. Concurrently, holes are being injected into $\mathrm{CuPc}$ from the AZO electrode. Due to a larger hole injection barrier for device A, the forward current remains low. As the forward bias is further increased, holes begin to overcome the injection barrier, driving a forward current to complete the sigmoidal $J-V$ characteristic.

In order to demonstrate ozone-treated AZO as an ITO alternative, devices employing AZO (device C) and ITO (device $\mathrm{D}$ ) anodes were fabricated in a single deposition sequence. Figure 4 shows $J-V$ characteristics of devices $C$ and D. Approximately equivalent performance was achieved by devices $\mathrm{C}$ and $\mathrm{D}$, with power conversion efficiencies matching within $10 \%$. The measured short circuit currents were nearly identical, from which we may conclude that there was little change in the optical field distribution, absorption of light in the active region, or in charge collection efficiency. Interestingly, devices made with AZO exhibited higher open circuit voltages than did those made with ITO. Table I compares relevant performance parameters for all devices. Reciprocal slopes of $J-V$ relations at $V_{\mathrm{OC}}$ are used to compare the series resistances, $R_{S}$, of the devices. ${ }^{13}$

In summary, we have shown that ozone treated AZO is an effective ITO alternative material for $\mathrm{CuPc}$-based organic photovoltaics. AZO was found to vary considerably with target-to-substrate Treating AZO films with UV-generated ozone resulted in improved conductivity. OPV cells fabricated on ozone treated AZO outperformed cells fabricated on as-deposited AZO, showing higher $V_{\mathrm{OC}}$, fill factor (FF), and power conversion efficiency. These improvements were explained through band alignment under $V_{\mathrm{OC}}$ conditions employing quasi-Fermi-level splitting.

${ }^{1}$ C. Tang, Appl. Phys. Lett. 48, 183 (1986).

${ }^{2}$ J. Xue, B. P. Rand, S. Uchida, and S. R. Forrest, Appl. Phys. Lett. 85, 5757 (2004)

${ }^{3}$ J. Y. Kim, K. Lee, N. E. Coates, D. Moses, T. Nguyey, M. Dante, and A.

J. Heeger, Science 317, 222 (2007).

${ }^{4}$ V. Bosle, J. T. Prater, F. Yang, D. Burk, S. R. Forrest, and J. Narayan, J. Appl. Phys. 102, 023501 (2007).

${ }^{5}$ A. V. Singh, N. Buthrath, A. Wakahara, A. Yoshida, and R. M. Mehra, J. Appl. Phys. 90, 5661 (2001).

${ }^{6}$ J. C. Bernede, Y. Berredjem, L. Cattin, and M. Morsli, Appl. Phys. Lett. 92, 083304 (2008).

${ }^{7}$ K. Schulze, B. Maennig, Y. Tomita, C. May, J. Hüpkes, E. Brier, E. Reinold, P. Bäuerle, and K. Leo, Appl. Phys. Lett. 91, 073521 (2007).

${ }^{8}$ A. V. Singh, R. M. Mehra, A. Yoshida, and A. Wakahara, J. Appl. Phys. 95, 3640 (2004).

${ }^{9}$ P. Peumans and S. R. Forrest, Appl. Phys. Lett. 79, 126 (2001).

${ }^{10}$ C. M. Ramsdale, J. A. Barker, A. C. Arias, J. D. MacKenzie, R. H. Friend, and N. C. Greenham, J. Appl. Phys. 92, 4266 (2002).

${ }^{11}$ B. Gregg, J. Phys. Chem. B 107, 4688 (2003).

${ }^{12}$ C. Uhrich, D. Wynands, S. Olthof, M. K. Riede, K. Leo, S. Sonntag, B. Maennig, and M. Pfeiffer, J. Appl. Phys. 104, 043107 (2008).

${ }^{13}$ B. Brousse, B. Ratier, and A. Moliton, Synth. Met. 147, 293 (2004). 